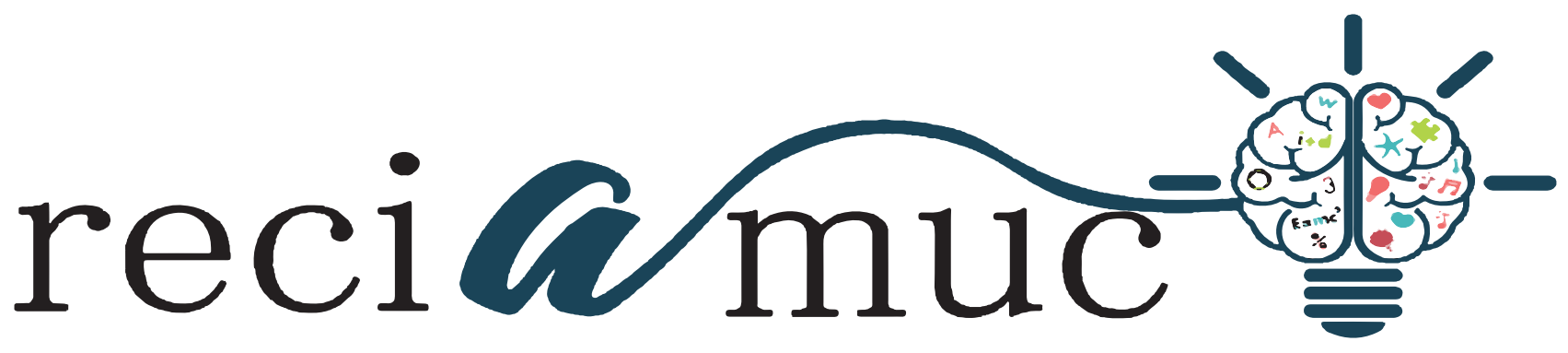

DOI: 10.26820/reciamuc/5.(1).ene.2021.140-147

URL: https://reciamuc.com/index.php/RECIAMUC/article/view/602

EDITORIAL: Saberes del Conocimiento

REVISTA: RECIAMUC

ISSN: 2588-0748

TIPO DE INVESTIGACIÓN: Artículo de Revisión

CóDIGO UNESCO: 32 Ciencias Médicas

PAGINAS: $140-147$

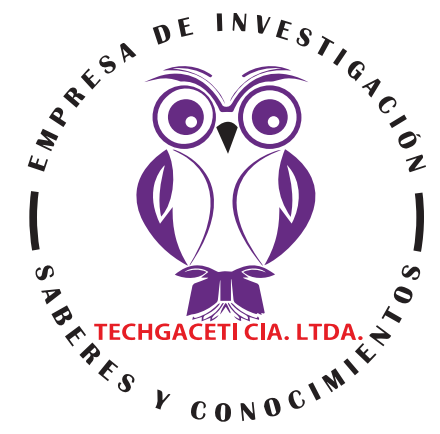

\title{
Diagnóstico y tratamiento de prolactinoma
}

Diagnosis and treatment of prolactinoma

Diagnóstico e tratamento do prolactinoma

\section{Karen Michelle Ruilova Gavilanes'; Tessa Katherina Vela López2; Ivonne Paulette Bravo Galarza3; Luis Angel Medina Idrovo ${ }^{4}$}

RECIBIDO: 10/11/2020 ACEPTADO: 29/11/2020 PUBLICADO: 31/01/2021

1. Médico Residente; Quito, Ecuador; krenmr@gmail.con; D https://orcid.org/0000-0002-9314-4366

2. Médico General; Investigadora Independiente; Quito, Ecuador; tessakathvela@hotmail.com; (D) https://orcid. org/0000-0003-0184-9156

3. Médica Residente; Quito, Ecuador; paubravo02@gmail.com; (D) https://orcid.org/0000-0002-1621-077X

4. Médico; Investigador Independiente; Cuenca, Ecuador; Imedinaidrovo@gmail.com; (D) https://orcid.org/00000001-7851-2856

\section{CORRESPONDENCIA}

Karen Michelle Ruilova Gavilanes

ellen1689@hotmail.com

$$
\text { Quito, Ecuador }
$$

(C) RECIAMUC; Editorial Saberes del Conocimiento, 2021 


\section{RESUMEN}

Los tumores hipofisarios benignos y pequeños son bastante comunes en la población mundial. Entre ellos, el prolactinoma es el tipo más común de tumor hipofisario, el cual representa aproximadamente el $40 \%$ de todos los tumores hipofisarios. Un prolactinoma "es un tumor benigno (no canceroso) de la hipófisis, que produce una hormona llamada prolactina. Su prevalencia es de aproximadamente 500 casos por millón de habitantes y su incidencia es de 27 casos por millón por año. Entre sus principales complicaciones se encuentran osteoporosis, complicaciones asociadas al embarazo, reducción de los niveles de otras hormonas pituitarias y pérdida de la visión. El objetivo del presente estudio consiste en plasmar las generalidades relacionadas con el diagnóstico y el tratamiento básico del prolactinoma. El modelo de investigación es una revisión de tipo documental bibliográfico. Los prolactinomas son tumores muy frecuentes en la población mundial. La mayoría tienen muy buen pronóstico, no obstante, su diagnóstico temprano y preciso es necesario para la intervención terapéutica más favorable. El diagnóstico incluye evaluación de la hiperprolactinemia, la exclusión de otras causas de hiperprolactinemia (diagnóstico diferencial) y la realización de estudios de imagen. Mientras que el tratamiento general incluye en primera línea agonistas de la dopamina tales como: cabergolina y bromocriptina. En segunda línea se encuentran la Cirugía o procedimiento quirúrgico. Por último, como ratamiento ocasional, la Radioterapia. Asimismo, es importante destacar que el tratamiento dependerá del tamaño del tumor y de los síntomas ocasionados por la variación o exceso de niveles hormonales. Incluso algunos pacientes requieren la combinación de cirugía y radioterapia. En consecuencia, resulta fundamental un buen diagnóstico para intervenir adecuadamente el prolactinoma y obtener el mejor resultado para la salud de estos pacientes.

Palabras clave: Diagnóstico, tratamiento, prolactinoma, tumor, hipófisis.

\section{ABSTRACT}

Small, benign pituitary tumors are quite common in the world population. Among them, prolactinoma is the most common type of pituitary tumor, accounting for approximately $40 \%$ of all pituitary tumors. A prolactinoma "is a benign (non-cancerous) tumor of the pituitary gland, which produces a hormone called prolactin. Its prevalence is approximately 500 cases per million inhabitants and its incidence is 27 cases per million per year. Among its main complications are osteoporosis, complications associated with pregnancy, reduced levels of other pituitary hormones and loss of vision. The objective of this study is to capture the generalities related to the diagnosis and basic treatment of prolactinoma. The research model is a bibliographic documentary type review. Prolactinomas are very common tumors in the world population. Most have a very good prognosis, however, their accurate diagnosis is necessary for the most favorable early therapeutic intervention. Diagnosis includes evaluation of hyperprolactinemia, exclusion of other causes of hyperprolactinemia (differential diagnosis), and imaging. While the general treatment includes first-line dopamine agonists such as: cabergoline and bromocriptine. Second line is surgery or surgical procedure. Finally, as an occasional treatment, Radiotherapy. Likewise, it is important to highlight that the treatment depends on the size of the tumor and the symptoms caused by the variation or excess of hormonal levels. Some patients even need the combination of surgery and radiation therapy. Consequently, a good diagnosis is essential to properly intervene the prolactinoma and obtain the best result for the health of these patients.

KeyWords: Diagnosis, treatment, prolactinoma, tumor, pituitary.

\section{RESUMO}

Os pequenos e benignos tumores da hipófise são bastante comuns na população mundial. Entre eles, o prolactinoma é o tipo mais comum de tumor pituitário, representando aproximadamente $40 \%$ de todos os tumores pituitários. Um prolactinoma "é um tumor benigno (não cancerígeno) da hipófise, que produz uma hormona chamada prolactino. A sua prevalência é de aproximadamente 500 casos por milhão de habitantes e a sua incidência é de 27 casos por milhão por ano. Entre as suas principais complicações estão a osteoporose, complicações associadas à gravidez, níveis reduzidos de outras hormonas da hipófise e perda de visão. O objectivo deste estudo é captar as generalidades relacionadas com o diagnóstico e tratamento básico do prolactinoma. O modelo de investigação é uma revisão bibliográfica de tipo documental. Os prolactinomas são tumores muito comuns na população mundial. A maioria tem um prognóstico muito bom, no entanto, o seu diagnóstico preciso é necessário para a intervenção terapêutica precoce mais favorável. O diagnóstico inclui a avaliação da hiperprolactinemia, exclusão de outras causas de hiperprolactinemia (diagnóstico diferencial), e imagiologia. Enquanto o tratamento geral inclui agonistas dopaminérgicos de primeira linha, tais como: cabergolina e bromocriptina. A segunda linha é a cirurgia ou procedimento cirúrgico. Finalmente, como tratamento ocasional, a Radioterapia. Do mesmo modo, é importante salientar que o tratamento depende do tamanho do tumor e dos sintomas causados pela variação ou excesso de níveis hormonais. Alguns pacientes precisam mesmo da combinação de cirurgia e radioterapia. Consequentemente, um bom diagnóstico é essencial para intervir adequadamente no prolactinoma e obter o melhor resultado para a saúde destes pacientes.

Palavras-chave: Diagnóstico, tratamento, prolactinoma, tumor, pituitária. 


\section{Introducción}

Los tumores hipofisarios benignos y pequeños son bastante comunes en la población mundial. Entre ellos, el prolactinoma es el tipo más común de tumor hipofisario, el cual representa aproximadamente el $40 \%$ de todos los tumores hipofisarios. Un prolactinoma "es un tumor benigno (no canceroso) de la hipófisis, que produce una hormona llamada prolactina. La hipófisis (o pituitaria), ubicada en la base del cerebro, es una glándula del tamaño de un guisante que controla la producción de muchas hormonas". (Instituto Nacional de la Diabetes y las Enfermedades Digestivas y Renales de los Estados Unidos - NIDDK, 2019)

Entre las complicaciones del prolactinoma se pueden destacar las siguientes:

- Pérdida de la masa ósea (osteoporosis). El exceso de prolactina puede reducir la producción de las hormonas estrógeno y testosterona, de modo que disminuirá la densidad ósea y aumentará el riesgo de tener osteoporosis.

- Complicaciones en el embarazo. Durante un embarazo normal, aumenta la producción de estrógeno. Si estás embarazada y tienes un prolactinoma de gran tamaño, estos niveles elevados de estrógeno pueden causar un crecimiento del tumor y signos y síntomas asociados, como dolores de cabeza y cambios en la visión.

- Pérdida de la visión. Si el prolactinoma no se trata, puede agrandarse lo suficiente como para comprimir el nervio óptico. Esto puede causar una pérdida de visión periférica.

- Niveles bajos de otras hormonas pituitarias. Con prolactinomas más grandes, la presión sobre la glándula pituitaria normal puede disminuir los niveles de otras hormonas controladas por la glándula pituitaria, como las hormonas tiroideas y el cortisol (una hormona de respuesta al estrés). (Clínica Mayo, 2020)

Melgar et al., (2016) refiere que su prevalencia es de aproximadamente 500 casos por millón de habitantes y su incidencia es de 27 casos por millón por año. Asimismo, manifiesta:

Los prolactinomas se clasifican de acuerdo con su tamaño en microprolactinomas si son menores a $10 \mathrm{~mm}$, macroprolactinomas si son mayores a $10 \mathrm{~mm}$ y gigantes si son mayores a $40 \mathrm{~mm}$. El $70 \%$ de los tumores ocurre en mujeres y el $64 \%$ son microprolactinomas (con una razón mujer a hombre de 20 a 1). Los macroprolactinomas y los prolactinomas gigantes son más prevalentes en los hombres (proporción macro a microprolactinoma de 5 a 1). (p. 116)

El pronóstico por lo general es excelente, no obstante, depende del éxito de la terapia médica o de la cirugía. Resulta fundamental realizar exámenes para verificar si el tumor ha reaparecido después del tratamiento. Igualmente, es importante destacar que, el tratamiento para el prolactinoma puede cambiar los niveles de otras hormonas en el cuerpo, especialmente si se realiza cirugía o radiación. Los niveles altos de estrógeno o testosterona pueden estar involucrados en el crecimiento de un prolactinoma. A las mujeres con prolactinomas se les debe hacer un seguimiento cuidadoso durante el embarazo. Ellas deben hablar respecto a este tumor con su proveedor antes de tomar píldoras anticonceptivas con un contenido de estrógeno mayor de lo normal. (Enciclopedia Médica ADAM, 2019)

En virtud de lo cual, resulta fundamental el conocimiento científico que se pueda generar acerca del diagnóstico, así como del tratamiento de estos tumores hipofisarios, con la finalidad de reducir los índices de complicaciones que se puedan presentar.

El objetivo del presente estudio consiste en plasmar las generalidades relacionadas con el diagnóstico y el tratamiento básico del prolactinoma. 


\section{Materiales y métodos}

Para el desarrollo del presente estudio fueron ubicados una serie de archivos digitalizados, cuya búsqueda de contenidos científicos se llevó a cabo durante el mes de enero de 2021. Dicha metodología lo ubica como un estudio de tipo documental bibliográfico.

Se formularon ecuaciones de búsqueda, con palabras clave, operadores lógicos y booleanos, en determinadas bases de datos y/o buscadores especializados, tales como: SciELO, Researchgate, Medigraphic, Elsevier, entre otros. Asimismo, algunas páginas web relacionadas con el área de la salud y con amplio reconocimiento científico internacional tales como: MedlinePlus, Clínica Mayo, Instituto Nacional del Cáncer de los Estados Unidos, entre otros. Algunas de las formulaciones y descriptores utilizados con los que se obtuvieron mejores resultados fueron los siguientes: "diagnóstico de prolactinoma"; "prolactinoma"; "tratamiento del prolactinoma". La información obtenida fue filtrada bajo los criterios de idioma (español); disponibilidad del contenido (completo); periodo de publicación (2013-2021), tipo de estudio: revisiones sistemáticas, de cohorte y de casos o de controles, informe de casos; $y$, clase de material bibliográfico (consensos, manuales, ensayos, tesis de grado, posgrado o doctorado, informes y otras clases de contenidos).

Asimismo, se seleccionaron los recursos antes mencionados en base a su producción, aval o promoción por parte de instituciones, entes, organizaciones, sociedades o asociaciones de profesionales en el área de la salud, de carácter público o privado, nacionales, internacionales o multilaterales con reconocimiento científico dentro de la comunidad de la salud. Por último, fueron desestimados aquellos contenidos repetidos (duplicados), editoriales, anotaciones académicas y otros tipos de materiales bibliográficos de escaso valor científico, con bajo nivel de evidencia o aportado por tra- tadistas sin acreditación en el área de la salud o medicina.

\section{Resultados}

\section{Diagnóstico}

El diagnóstico del prolactinoma está basado en la evaluación de la hiperprolactinemia, la exclusión de otras causas de hiperprolactinemia (diagnóstico diferencial) y la realización de estudios de imagen. (Blanco, Fernández, \& Escribano, 2016, p. 842)

\section{Prueba de niveles de prolactina}

La prueba de niveles de prolactina, también llamada prueba de PRL test o análisis de sangre de prolactina, mide los niveles de prolactina $(P R L)$ en la sangre. La prolactina "es una hormona producida por la glándula pituitaria o hipófisis, una glándula pequeña situada en la base del cerebro. La prolactina hace que los senos crezcan y produzcan leche materna durante el embarazo y después del parto". Son normales niveles altos de prolactina en mujeres embarazadas y en periodo de lactancia. Los niveles son normalmente bajos para las mujeres no embarazadas y los hombres. Si los niveles de prolactina están más altos de lo normal, en general puede significar que hay un tumor de la glándula pituitaria conocido como prolactinoma. (Enciclopedia Médica ADAM, 2020)

\section{Diagnóstico diferencial de la hiperprolac- tinemia}

Para Blanco, Fernández, \& Escribano, (2016) "ante el hallazgo de hiperprolactinemia, deben excluirse causas fisiológicas, farmacológicas y patológicas de elevación de la misma" (ver Tabla 1). En tal sentido, se recomienda realizar pruebas de función hepática, renal y función tiroidea en todos los pacientes con hiperprolactinemia, y descartar el embarazo en mujeres en edad fértil. (p. 842) 
Tabla 1. Causas de hiperprolactinemia

\begin{tabular}{|c|c|c|c|c|c|}
\hline Causas & Causas. & \multicolumn{4}{|c|}{ Causas patológicas } \\
\hline Embarazo & $\begin{array}{l}\text { Antipsicóticos } 0 \\
\text { neurolépticos }\end{array}$ & $\begin{array}{l}\text { Enfermedades } \\
\text { hipotalámicas }\end{array}$ & $\begin{array}{c}\text { Enfermedades } \\
\text { hipofisiarias }\end{array}$ & $\begin{array}{c}\text { Patología } \\
\text { endocrinológica }\end{array}$ & Otras \\
\hline Lactancia & Antidepresivos & $\begin{array}{l}\text { Craneofaringioma } \\
\text { meningioma y } \\
\text { linfoma }\end{array}$ & Prolactinoma & $\begin{array}{l}\text { Síndrome de } \\
\text { ovario } \\
\text { poliquístico }\end{array}$ & $\begin{array}{l}\text { Insuficiencia } \\
\text { renal }\end{array}$ \\
\hline $\begin{array}{l}\text { Estímulo del } \\
\text { pezóncicio }\end{array}$ & Antihipertensivos & $\begin{array}{l}\text { Sarcoidosis, } \\
\text { histiocitosis X }\end{array}$ & $\begin{array}{l}\text { Tumores } \\
\text { mixtos } \\
\text { (GH/PRL) }\end{array}$ & $\begin{array}{l}\text { Hipotiroidismo } \\
\text { primario }\end{array}$ & $\begin{array}{l}\text { Cirrosis } \\
\text { hepática }\end{array}$ \\
\hline $\begin{array}{l}\text { Estrés físico } \\
\text { o psicológico }\end{array}$ & Antieméticos & Sección del tallo & $\begin{array}{l}\text { Adenomas no } \\
\text { funcionantes }\end{array}$ & & $\begin{array}{l}\text { Lesión en } \\
\text { pared torácica }\end{array}$ \\
\hline $\begin{array}{l}\text { Ejercicio } \\
\text { físico }\end{array}$ & Opiáceos & Irradiación & $\begin{array}{l}\text { Silla turca } \\
\text { vacía }\end{array}$ & & $\begin{array}{l}\text { Lesión } \\
\text { medular }\end{array}$ \\
\hline & $\begin{array}{l}\text { Otros: } \\
\text { estrógenos, } \\
\text { marihuana }\end{array}$ & $\begin{array}{l}\text { Lesiones } \\
\text { vasculares }\end{array}$ & $\begin{array}{l}\text { Hipófisitis } \\
\text { linfocítica }\end{array}$ & & \\
\hline & & $\begin{array}{l}\text { Traumatismo } \\
\text { craneoencefálico }\end{array}$ & & & \\
\hline
\end{tabular}

Fuente: GH: hormona del crecimiento; PRL: prolactina. Modificado de: Blanco, Fernández, \& Escribano, (2016).

\section{Imágenes}

Halperin, Cámara, García, \& Ollero, (2013) refieren con relación a esta otra prueba diagnóstica del prolactinoma, lo siguiente:

La prueba de imagen indicada para el estudio de lesiones hipofisarias es la resonancia magnética (RM). La exploración puede realizarse tras detectar una hiperprolactinemia en la que se han excluido las causas secundarias, o indicarse por otros motivos (estudio de cefalea, alteraciones campimétricas, etc.), con hallazgo de la lesión hipofisaria, y posterior estudio funcional diagnóstico de hiperprolactinemia. Se recomienda estudio de imágenes potenciadas en T1 y T2, y tras la inyección de contraste paramagnético (gadolinio), con cortes finos (2-3 mm). Si no es posible esta exploración, deberá practicarse una tomografía computarizada de alta definición. Si se tiene en cuenta que la conducta inicial frente a un microprolactinoma y una hiperprolactinemia idiopática persistente será similar, no se plantea una búsqueda más intensiva de microadenomas cuando no son evidentes con esta exploración. No se han valorado, por lo tanto,
RM de 3 teslas, tal como se ha estudiado en otros microadenomas en los que de todos modos no mejoró la sensibilidad de localización. Tampoco se ha valorado de forma sistemática la tomografía de emisión de positrones con diversos trazadores como FDG, C11- metionina o F18- dopa12. (p. 312)

\section{Tratamiento}

Las opciones de tratamiento estándar para los tumores de hipófisis productores de PRL incluyen: "Agonistas de la dopamina tales como cabergolina y bromocriptina. Cirugía (segunda línea). Radioterapia (ocasional)". Cuando el tumor de hipófisis segrega $P R L$, el tratamiento dependerá del tamaño del tumor y de los síntomas que ocasiona una producción hormonal excesiva. Los pacientes con tumores secretores de PRL se tratan con cirugía y radioterapia. (Instituto Nacional del Cáncer de los Estados Unidos, 2020)

\section{Agonistas de la dopamina}

El tratamiento de elección para el prolactinoma son los agonistas dopaminérgicos. Al respecto, Salazar et al., (2014) refieren lo 
siguiente:

Los fármacos más indicados y con los que se cuenta mayor experiencia clínica son la cabergolina y la bromocriptina. La evidencia de estudios comparativos entre bromocriptina y cabergolina apoya de manera convincente la superioridad de la cabergolina en términos de tolerabilidad, conveniencia, reducción de las concentraciones de prolactina sérica, restitución de la función gonadal y disminución del tamaño del prolactinoma. Además, la cabergolina es de gran utilidad en pacientes resistentes a la bromocriptina. Sin embargo, la bromocriptina es un fármaco que se ha prescrito de manera satisfactoria durante muchos años, quizá debido a que su costo-beneficio (porque ha sido estudiada por más tiempo que la cabergolina) supera los efectos secundarios. (p. 136, 137)

La cabergolina es un agonista selectivo de receptores D2 de la dopamina cuya eficacia y tolerabilidad han sido ampliamente demostradas. Utilizando dosis de 0.25 a 3 mg a la semana se logra la normoprolactinemia y la reducción significativa del tamaño tumoral en aproximadamente 91\% de los pacientes con microprolactinomas y $77 \%$ con macroprolactinomas. En cuanto a eficacia, con la bromocriptina (otro agonista dopaminérgico) se logra la normoprolactinemia en $80 \%$ de microprolactinomas y en $70 \%$ de macroprolactinomas, solo que con una mayor frecuencia de efectos adversos, sobre todo gastrointestinales. En un 10 a $15 \%$ de pacientes tratados con cabergolina no se logra la normoprolactinemia y estos pacientes son considerados resistentes a agonistas dopaminérgicos. (Melgar et al., 2016, p. 118)

\section{Cirugía}

Alrededor de $10 \%$ de los pacientes con macroadenomas hipofisarios no responderán o serán resistentes al tratamiento con agonistas dopaminérgicos. Es en este grupo de pacientes donde deben valorarse otras opciones de tratamiento, como el quirúrgico.
Salazar et al., (2014) explicacal respecto:

Las indicaciones de abordaje quirúgico de los macroadenomas incluyen: falla o resistencia a la terapia farmacológica, apoplejía hipofisaria con síntomas de daño neurológico y los macroadenomas hipofisarios quísticos que regularmente no responden al tratamiento con agonistas dopaminérgicos. La cirugía transesfenoidal no es curativa en un porcentaje importante de casos, y la recurrencia de la hiperprolactinemia es frecuente. Por ello es necesario informar y discutir con el paciente las ventajas de la cirugía y del tratamiento crónico con agonistas dopaminérgicos y con base en ello la decisión del paciente se considerará criterio de tratamiento quirúgico. (p. 138)

El abordaje transesfenoidal microquirúrigico, tanto por vía sublabial como por vía transnasal, es el más utilizado. En los últimos años, el abordaje transesfenoidal por vía transnasal endoscópico, ha irrumpido como la técnica de elección para el abordaje de las lesiones de la región selar, con idénticos resultados y recuperaciones más rápidas. El abordaje transesfenoidal transnasal "es un tipo de cirugía en la que se introducen instrumentos (endoscopio y curetas) a través de las narinas o fosas nasales y el seno esfenoidal para acceder a la hipófisis y extraer tumores presentes en la glándula o cerca de ella" (ver Figura 1). Asimismo, la autora citando Tortosa y Webb, (2017) explica lo siguiente:

El abordaje transesfenoidal por vía transnasal endoscópico presenta ventajas sobre todo en tumores de gran tamaño y con extensión lateral. Desde su aparición hace aproximadamente unos 10 años, se ha impuesto como la alternativa quirúrgica presentando una mayor tasa de éxeresis tumoral (extirpación), mejor control de la enfermedad y una menor tasa de complicaciones. (Martín Cabrera, 2018, p. 21, 22) 

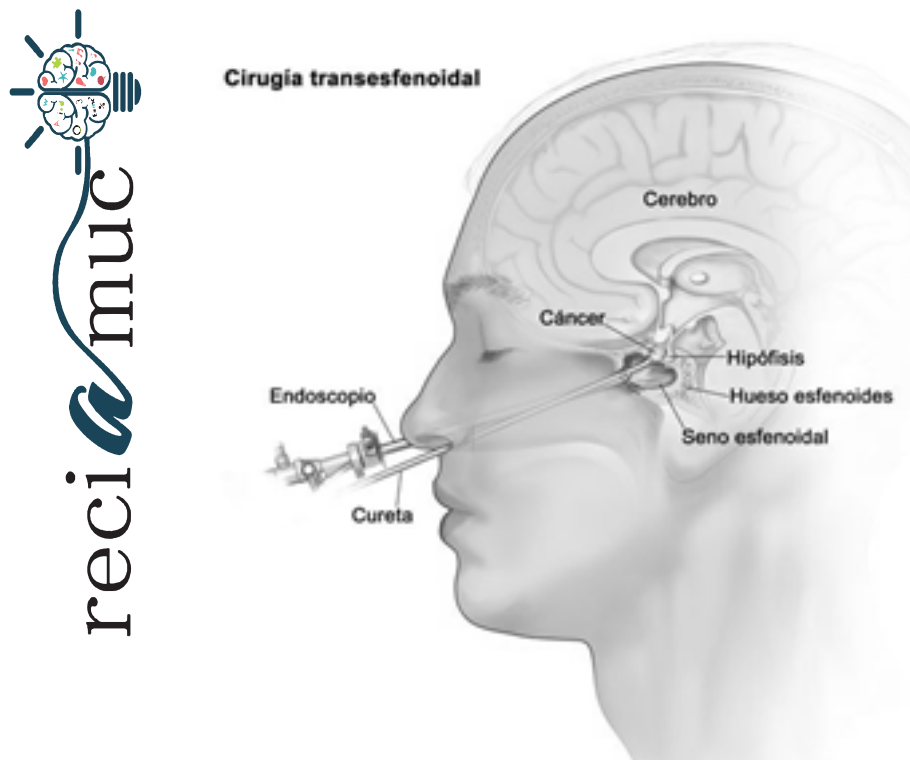

Figura 1. Representación esquemática en visión lateral en la que se muestra la técnica quirúrgica de abordaje transesfenoidal transnasal para la extirpación de tumores hipofisarios

Fuente: Instituto Nacional del Cáncer de EE.UU. por Martín Cabrera, P. (2018). "Biología clínica y abordaje terapéutico de los adenomas hipofisarios". Universidad de La Laguna. p. 22. URL: https://riull.ull.es/xmlui/bitstream/handle/915/9693/Biologia,\%20clinica\%20y\%20 abordaje\%20terapeutico\%20de\%20adenomas\%20hipofisarios.. pdf; jsessionid=FB68791026648ED6931B8412B860CCFO? sequence $=1$

\section{Radioterapia}

La radioterapia, como alternativa de tratamiento de los macroadenomas, es muy poco utilizada dada la frecuencia de sus efectos colaterales, como el hipopituitarismo, daño al nervio óptico, disfunción neurológica e incremento del riesgo tumores cerebrales, las cuales son altas. "La radioterapia puede ser una opción en pacientes con macroadenomas que no responden a los agonistas dopaminérgicos, con recurrencia posterior a la cirugía y específicamente en los casos sumamente raros de prolactinomas malignos". (Salazar et al., 2014, p. 138)
Rojas, (2017) agrega acerca de la radioterapia las siguientes consideraciones:

La radioterapia es extremadamente útil si luego de una o más cirugías queda remanente tumoral. Existe evidencia de Nivel 2 que apoya el uso de radioterapia o radiocirugía para manejo del remanente tumoral. En estos casos, se recomienda el uso de dosis 12 Gy en el caso de la radiocirugía o dosis fraccionadas de 45-54 G y para lograr control local del tumor sobre un $90 \%$ a 5 años. Esto es especialmente útil en que pacientes en que el riesgo de una reintervención se considere elevado debido a la edad o condiciones médicas asociadas (Nivel 3). En el caso de que no quede remanente tumoral después de la resección o el remanente sea pequeño, se recomienda seguimiento con imágenes (Nivel 2). (p. 417)

\section{Conclusiones}

Los prolactinomas son tumores muy frecuentes en la población mundial. La mayoría tienen muy buen pronóstico, no obstante, su diagnóstico temprano y preciso es necesario para la intervención terapéutica más favorable.

El diagnóstico incluye evaluación de la hiperprolactinemia, la exclusión de otras causas de hiperprolactinemia (diagnóstico diferencial) y la realización de estudios de imagen.

Mientras que el tratamiento general incluye en primera línea agonistas de la dopamina tales como: cabergolina y bromocriptina. En segunda línea se encuentran la Cirugía o procedimiento quirúrgico. Por último, como ratamiento ocasional, la Radioterapia. Asimismo, es importante destacar que el tratamiento dependerá del tamaño del tumor y de los síntomas ocasionados por la variación o exceso de niveles hormonales. Incluso algunos pacientes requieren la combinación de cirugía y radioterapia. En consecuencia, resulta fundamental un buen diagnóstico para intervenir adecuadamente 
el prolactinoma y obtener el mejor resultado para la salud de estos pacientes.

\section{Bibliografía}

Blanco, C., Fernández, C., \& Escribano, P. (2016). Adenomas funcionantes de la hipófisis. Medicine, 12(15), 838-849. Recuperado el 20 de Enero de 2021, de http://residenciamflapaz.com/Articulos\%20Residencia\%2017/126\%20Adenomas\%20 funcionantes\%20de\%20la\%20hip\%C3\%B3fisis. pdf

Clínica Mayo. (28 de Mayo de 2020). Clínica Mayo. Recuperado el 11 de Enero de 2021, de https://www. mayoclinic.org/es-es/diseases-conditions/prolactinoma/symptoms-causes/syc-20376958\#: :tex$\mathrm{t}=$ Este\%20tumor\%20hace\%20que\%20la,la\%20 testosterona\%20en\%20los\%20hombres.

Enciclopedia Médica ADAM. (06 de Mayo de 2019). MedlinePlus. Recuperado el 08 de Enero de 2021, de https://medlineplus.gov/spanish/ency/article/000336.htm

Enciclopedia Médica ADAM. (17 de Diciembre de 2020). MedlinePlus. Recuperado el 10 de Enero de 2021, de https://medlineplus.gov/spanish/pruebas-de-laboratorio/niveles-de-prolactina/

Halperin, I., Cámara, R., García, M., \& Ollero, D. (2013). Guía clínica de diagnóstico y tratamiento del prolactinoma y. Endocrinología y Nutrición, 60(6), 308-319. Recuperado el 24 de Enero de 2021, de https://www.elsevier.es/es-revista-endocrinologia-nutricion-12-pdf-S1575092213000296

Instituto Nacional de la Diabetes y las Enfermedades Digestivas y Renales de los Estados Unidos - NIDDK. (Septiembre de 2019). Institutos Nacionales de la Salud de los Estados Unidos. Recuperado el 09 de Enero de 2021, de https://www.niddk.nih. gov/health-information/informacion-de-la-salud/ enfermedades-endocrinas/prolactinoma
Instituto Nacional del Cáncer de los Estados Unidos. (08 de Diciembre de 2020). Instituto Nacional del Cáncer de los Estados Unidos. Recuperado el 11 de Enero de 2021, de https://www.cancer. gov/espanol/tipos/hipofisis/pro/tratamiento-hipofisis-pdq\#_123

Martín Cabrera, P. (2018). Biología clínica y abordaje terapéutico de los adenomas hipofisiarios. Tesis de grado, Universidad de La Laguna, Facultad de Ciencias. Recuperado el 21 de Enero de 2021, de https://riull.ull.es/xmlui/bitstream/handle/915/9693/ Biologia,\%20clinica\%20y\%20abordaje\%20terapeutico\%20de\%20a

Melgar, V., Espinosa, E., Sosa, E., Rangel, M. J., Cuenca, D., Ramírez, C., \& Mercado, M. (2016). Diagnóstico y tratamiento actual de la hiperprolactinemia. Revista Médica del Instituto Mexicano del Seguro Social, 54(1), 111-121. Recuperado el 16 de Enero de 2021, de https://www.redalyc.org/ pdf/4577/457745148023.pdf

Rojas, D. (2017). Manejo de los tumores de hipófisis. Revista Médica Clínica Condes, 28(3), 409-419. Recuperado el 20 de Enero de 2021, de 5BFEF2E5C6849A2951D14FD850604C50A55CBCBAD47F43E2906845CDE72B3A4DA726B2A34C4DDA31A6E9D73CE127D598

Salazar, C., Hernández, J., González, D., López, M., Ortíz, A., Porias, H., . . Vital, V. (2014). Guía de práctica clínica para. Ginecología y obstetricia de México, 82, 123-142. Recuperado el 28 de Enero de 2021, de https://www.medigraphic.com/pdfs/ ginobsmex/gom-2014/gom142g.pdf

\section{CITAR ESTE ARTICULO:}

Ruilova Gavilanes, K. M., Vela López, T. K., Bravo Galarza, I. P., \& Medina Idrovo, L. A. (2021). Diagnóstico y tratamiento de prolactinoma. RECIAMUC, 5(1), 140-147. https://doi.org/10.26820/reciamuc/5.(1).ene.2021.140-147 